\title{
Gabapentin reduces CX3CL1 signaling and blocks spinal microglial activation in monoarthritic rats
}

\author{
Jia-Le Yang ${ }^{1 \dagger}$, Bo Xu ${ }^{2 \dagger}$, Shuang-Shuang Li ${ }^{3}$, Wei-Shi Zhang ${ }^{3}$, Hua Xu ${ }^{3}$, Xiao-Ming Deng ${ }^{3}$ and Yu-Qiu Zhang ${ }^{1 *}$
}

\begin{abstract}
Background: Spinal glia, particularly microglia and astrocytes, are of the utmost importance in the development and maintenance of chronic pain. A recent study from our laboratory revealed that gabapentin, a recommended first-line treatment for multiple neuropathic conditions, could also efficiently antagonize thermal hyperalgesia evoked by complete Freund's adjuvant (CFA)-induced monoarthritis (MA). In the present study, we investigated whether the spinal glia are involved in the anti-hyperalgesic effect of gabapentin and how this event occurs.

Results: Unilateral intra-articular injection of CFA produced a robust activation of microglia and astrocytes. These cells exhibited large cell bodies, thick processes and increases in the ionized calcium binding adapter molecule 1 (Iba-1, a microglial marker) or the glia fibrillary acidic protein (GFAP, an astrocytic marker). These cells also displayed immunoreactive signals, and an upregulation of the voltage-gated calcium channels (VGCCs) a2/ $/ \delta-1$ subunit, CX3CL1 and CX3CR1 expression levels in the spinal cord. These changes were associated with the development of thermal hyperalgesia. Immunofluorescence staining showed that VGCC a2/ $\delta-1$ subunit, a proposed gabapentin target of action, was widely distributed in primary afferent fibers terminals and dorsal horn neurons. CX3CL1, a potential trigger to activate microglia, colocalized with VGCC a2/ $/-1$ subunits in the spinal dorsal horn. However, its receptor CX3CR1 was mainly expressed in the spinal microglia. Multiple intraperitoneal (i.p.) gabapentin injections $(100 \mathrm{mg} / \mathrm{kg}$, once daily for 4 days with the first injection $60 \mathrm{~min}$ before intra-articular CFA) suppressed the activation of spinal microglia, downregulated spinal VGCC a2/ $/-1$ subunits decreased CX3CL1 levels and blocked the development of thermal hyperalgesia in MA rats.

Conclusions: Here we provide the first evidence that gabapentin diminishes CX3CL1 signaling and spinal microglia activation induced by joint inflammation. We also show that the VGCC a2/ $\delta-1$ subunits might be involved in these events.
\end{abstract}

Keywords: a2/ $\delta$-1 subunit of Voltage-gated calcium channels, CX3CL1, CX3CR1, Gabapentin, Glial activation, Monoarthritis

\section{Background}

Rheumatoid arthritis (RA) is a systemic inflammatory disorder characterized by pain as the predominant clinical feature. Like other conditions that produce chronic inflammatory pain, arthritis is characterized by a heightened pain response to noxious (hyperalgesia) or innocuous (allodynia) stimulation and pain at rest (spontaneous

\footnotetext{
* Correspondence: yuqiuzhang@fudan.edu.cn

${ }^{\dagger}$ Equal contributors

'Institute of Neurobiology, Institutes of Brain Science and State Key Laboratory of Medical Neurobiology, Fudan University, Shanghai 200032, China

Full list of author information is available at the end of the article
}

pain). The inflammation of the joint causes an increased efficacy of synaptic transmission between the primary afferent fibers and the dorsal horn neurons (known as peripheral sensitization). This corresponds with the development of central sensitization, whereby neurons within the spinal cord also become hyperexcitable with an increased response to peripheral stimulation. It is well known that spinal glia, particularly microglia (CNS macrophages) and astrocytes, are of the utmost importance in the development and maintenance of chronic pain [1-3]. Following peripheral or central inflammation and damage, the spinal glia become activated. This activation produces changes in morphology and increases in the release of algesic substances (especially pro-

\section{Biomed Central}


inflammatory cytokines), which enhance pain transmission $[1,4,5]$. Activation of the spinal glia appears to correlate with the development and maintenance of behavioral hypersensitivity induced by spinal injury, peripheral nerve injury, formalin, zymosan, and complete Freund's adjuvant (CFA) [5-10]. A series of studies from our laboratory further demonstrated that disruption of glial function with fluorocitrate (a glial metabolic inhibitor) or minocycline (a microglial inhibitor) markedly blocked thermal hyperalgesia and mechanical allodynia in CFA-induced monoarthritic rats. This disruption was associated with the ability to suppress spinal glial activation [8-11], suggesting that activated glia play a major role in mediating the behavioral hypersensitivity evoked by joint inflammation.

Gabapentin, which has only minor side effects at clinically effective doses, has been widely used in the treatment of chronic pain states [12]. In animal studies, gabapentin has also been shown to possess analgesic properties in a wide range of chronic pain models including peripheral and central neuropathic, postsurgical, and arthritic pain [12-16]. Although the mechanisms of action of gabapentin have yet to be ascertained, evidence implies that gabapentin may act at the $\alpha 2 / \delta-1$ subunit of the calcium channel [17]. Gabapentin is thought to decrease neuronal activity by binding to the $\alpha 2 / \delta-1$ subunit. This binding likely inhibits calcium currents and prevents extracellular calcium entry, which is essential for subsequent vesicular exocytosis $[14,18,19]$. Also, evidence shows that gabapentin is an inhibitor of calcium channel subunit trafficking [20,21]. Additionally, gabapentin is able to block the persistent sodium current $\left(I_{\mathrm{NaP}}\right)$ [22] and open $\mathrm{K}^{+}$channels [23], inhibiting the abnormal spontaneous activity and hyperexcitability of sensory neurons, leading to a reduction of pain in rats. A recent report showed that oral gabapentin can also significantly reverse diabetes-induced allodynia and suppress microglial activation in the spinal dorsal horn. This evidence suggests that gabapentin may exert its anti-allodynic actions partially through alterations of microglia function [24]. In the present study, we further address whether the anti-hyperalgesic effect of gabapentin in CFA monoarthritic rats is also linked to a reduction of in the activation of spinal glia. If so, the possible mechanisms by which gabapentin modulates the activation of glia would be further defined.

\section{Results}

Time course of MA-induced spinal glial activation and thermal hyperalgesia

In agreement with our previous study [8-10], the present results show an early, robust microglial activation and a delayed activation of astrocytes in the spinal cord during the CFA-induced MA. After CFA-induced MA, robust microglial activation occurred in the ipsilateral lumbar spinal dorsal horn at 4 hrs. However, on days 1 and 4 after MA, activated microglia were observed bilaterally throughout the lumbar sections of the spinal cord. Activated microglia exhibited large cell bodies and short or thick processes. The ionized calcium binding adapter molecule 1 (Iba-1, a microglial marker) and the immunoreactive (Iba-1-IR) signals were increased in MA rats (Figures 1A and 2A). A one-way ANOVA analysis showed that the density of Iba-1-IR on both sides of the spinal cord was significantly greater in MA rats than that in the controls (ipsilateral: $F_{3,20}=48.598, p<0.01$; contralateral: $\mathrm{F}_{3,20}=19.557, \mathrm{p}<0.01$ ) (Figure $1 \mathrm{C}$ ). Unlike microglia, significant activation of the astrocytes in the spinal cord was observed 4 days after MA. Activated astrocytes exhibited intense glia fibrillary acidic protein (GFAP) immunoreactivity (GFAP-IR) and appeared to be hypertrophied with thick processes (Figures $1 \mathrm{~B}$ and $2 \mathrm{~B}$ ). A one-way ANOVA analysis showed that the density of GFAP-IR on both sides of the spinal cord was significantly greater in the MA rats compared to the controls (ipsilateral: $\quad \mathrm{F}_{3,20}=29.138, \quad \mathrm{p}<0.01 ; \quad$ contralateral: $\mathrm{F}_{3,20}=43.742, \mathrm{p}<0.01$ ) (Figure 1D).

The baseline measures of the paw withdrawal latency (PWL) to radiant heat stimulation did not differ in either hindpaw across the groups. As in our previous study [11], unilateral intra-articular injection of CFA produced marked joint inflammation (edema and erythema) and thermal hyperalgesia in the ipsilateral paw. This inflammation peaked at 1 day after CFA injection and showed little change over 6 days. Neither the PWLs of both hindpaws in the sham MA (intra-articular injection of NS) rats, nor the hindpaw contralateral to the CFAinjected joint had a significant difference before and after the intra-articular injection (Figure 1E).

\section{Effects of multiple i.p. gabapentin on spinal glial activation and the development of thermal hyperalgesia in CFA-induced monoarthritic rats}

When repeated i.p. injections of gabapentin $(100 \mathrm{mg} / \mathrm{kg})$ were given once daily for 4 successive days (with the initial injection 1 hour before intra-articular CFA), MAinduced spinal microglial activation was significantly suppressed by day 4 (One-way ANOVA, ipsilateral: $\mathrm{F}_{2,15}=66.27, \mathrm{q}=10.458, \mathrm{p}<0.01 ;$ contralateral: $\mathrm{F}_{2,15}=$ 31.67, $\mathrm{q}=4.950, \mathrm{p}<0.01$ ) (Figure $2 \mathrm{~A}$ and $\mathrm{C}$ ).

Repeated i.p. injections of gabapentin $(100 \mathrm{mg} / \mathrm{kg})$ caused a partial decrease in GFAP-IR on both sides of the spinal dorsal horn, although this did not reach statistical significance (One-way ANOVA, ipsilateral: $\mathrm{F}_{2,15}=48.29, \quad \mathrm{q}=3.003, \quad \mathrm{p}=0.051 ; \quad$ contralateral: $\mathrm{F}_{2,15}=42.076, \mathrm{q}=2.701 \mathrm{p}=0.076$ ) (Figure 2B and 2D).

Our previous study showed that a single i.p. injection of gabapentin $(25,50,100$, and $200 \mathrm{mg} / \mathrm{kg})$ dose- 

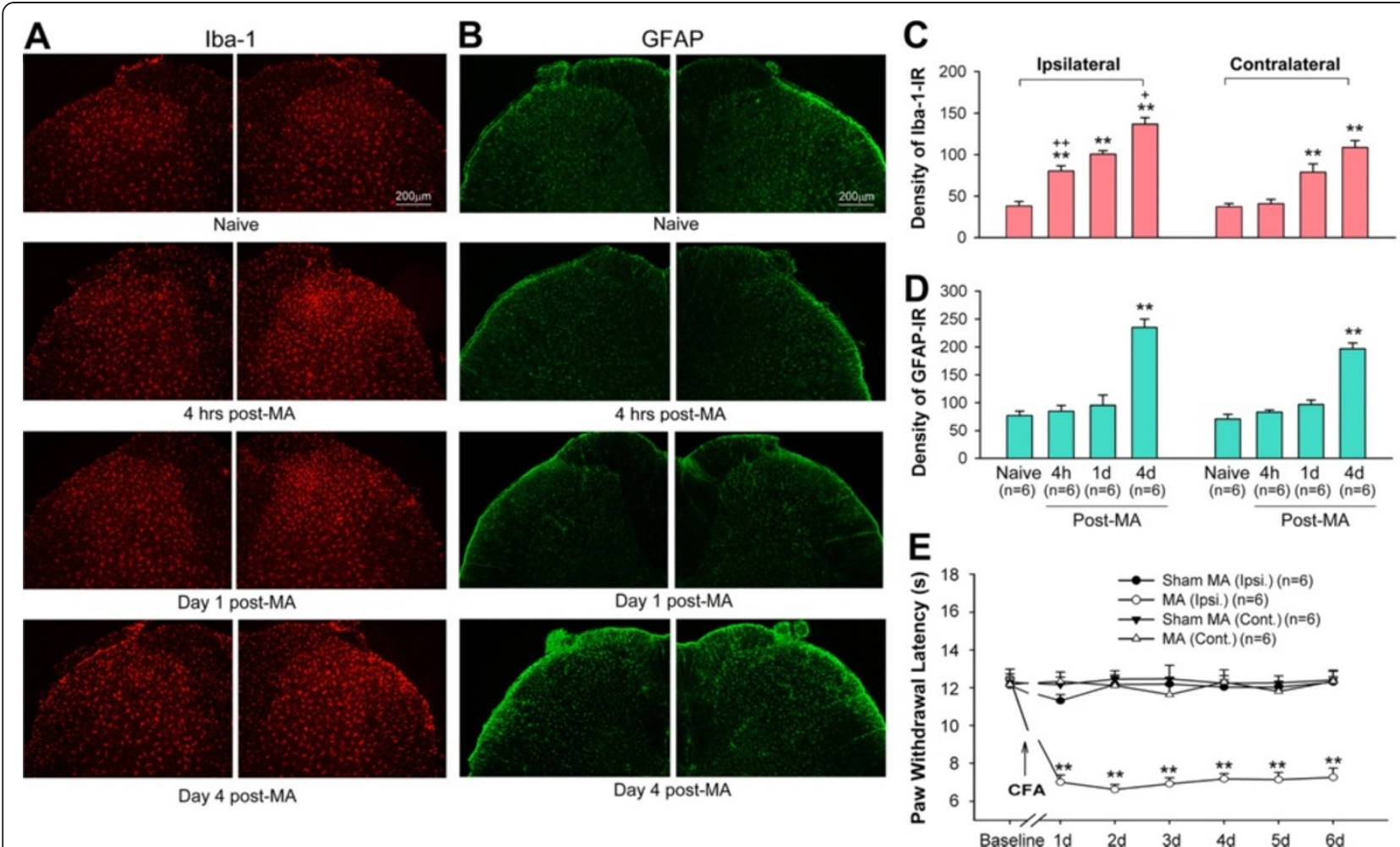

Figure 1 The activation of spinal glia (microglia and astrocytes) and thermal hyperalgesia by unilateral intra-articular injection of complete Freund's adjuvant (CFA)-induced monoarthritis (MA). A, Immunohistochemistry reveals an extensive increase in Iba-1- (a microglial marker) immunoreactivity (Iba-1-IR) in the ipsilateral lumbar spinal dorsal horn at 4 hrs and in both sides by days 1 and 4 after MA. B, Immunohistochemistry reveals a marked increase in GFAP- (a microglial marker) immunoreactivity (GFAP-IR) in both sides of the lumbar spinal dorsal horn on day 4 after MA. C and D, Quantification of Iba-1-IR (C) and GFAP-IR (D) expression on both sides of the spinal dorsal horn ** $p<0.01$ vs. naïve control; $+p<0.05,++p<0.01$ vs. contralateral. E, MA induced significant thermal hyperalgesia in the ipsilateral hindpaw ** $p<0.01$ vs. sham MA.

dependently reversed the established thermal hyperalgesia in MA rats [15]. A study from Dickenson's laboratory showed that chronic gabapentin treatment efficiently inhibited osteoarthritis-induced mechanical and cooling allodynia, as well as ambulatory-evoked pain [16]. To address the cumulative effects of gabapentin in the development of thermal hyperalgesia during arthritic pain, gabapentin $(100 \mathrm{mg} / \mathrm{kg})$ was repeatedly given once daily for 4 days (with the first injection $60 \mathrm{~min}$ before intra-articular CFA). The first injection of gabapentin before MA failed to prevent the development of thermal hyperalgesia that reliably occurred on day 1 post-MA. Following subsequent daily gabapentin administration, MA-induced ipsilateral hyperalgesia was significantly attenuated from day 2 to day 6 . Three days after cessation of the i.p. gabapentin, the PWLs were still reliably greater in gabapentin-treated animals than that in NS-treated animals (Figure 2E). A two-way ANOVA analysis revealed a significant effect of the gabapentin treatment $\left(\mathrm{F}_{1,14}=67.458, \mathrm{p}<0.01\right)$ and an interaction between the gabapentin treatment and time $\left(\mathrm{F}_{8,84}=6.847, \mathrm{p}<0.01\right)$.

\section{Expression of the VGCC $a 2 / \delta-1$ subunit in the spinal dorsal horn}

An increase in the VGCC $\alpha 2 / \delta-1$ subunit (an analgesic gabapentin target of action), in the dorsal spinal cord has been reported in diabetic- and paclitaxel-induced pain $[17,25]$. Consistent with this observation, the present study showed a significant increase in the expression of the VGCC $\alpha 2 / \delta-1$ subunit in the ipsilateral spinal dorsal horn at $4 \mathrm{hrs}$ and 4 days after CFA-induced MA (Figure 3A). After repeated i.p. injections of gabapentin $(100 \mathrm{mg} / \mathrm{kg}$, given once daily for 4 successive days with the initial injection 1 hour before intra-articular CFA), MA-induced upregulation of the VGCC $\alpha 2 / \delta-1$ subunit was significantly suppressed by day 4 post-MA (One-way ANOVA, $\quad \mathrm{F}_{2,9}=18.44, \quad \mathrm{q}=7.87, \quad \mathrm{p}<0.01$ ) (Figure 3B). Moreover, we examined whether the VGCC $\alpha 2 / \delta-1$ subunit is, co-expressed with Iba- 1 and GFAP in the spinal dorsal horn. This experiment was to determine the possible mechanisms of gabapentin-induced inhibition in the activation of spinal glia. Unexpectedly, we found no evidence that the VGCC $\alpha 2 / \delta-1$ subunit colocalized with the microglial marker Iba-1 or the 


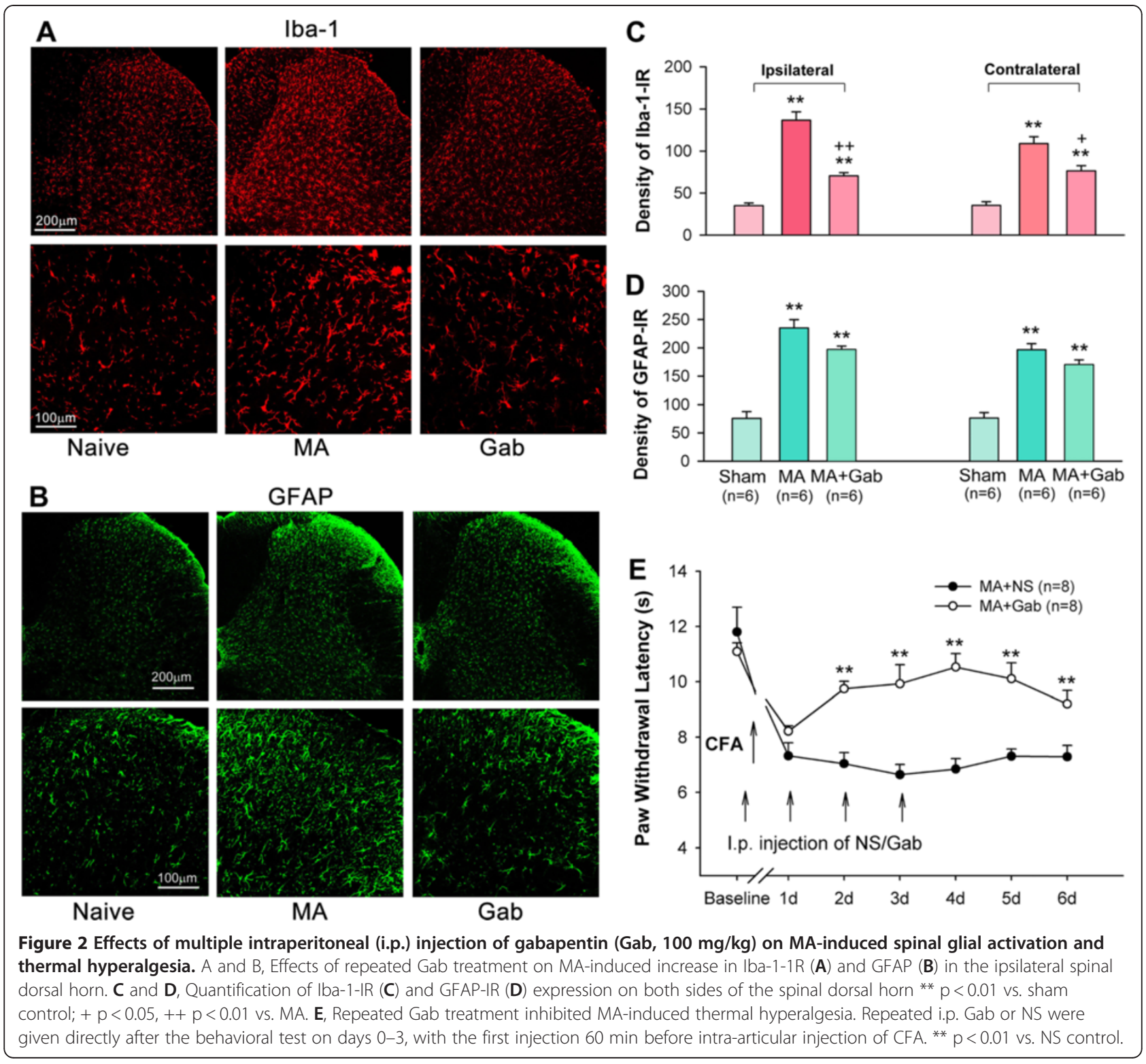

astrocytic marker GFAP in the spinal dorsal horn (Figure $3 C$ ). In contrast, the $\alpha 2 / \delta$ - 1 -IR signals were mainly detected in the primary afferent termination of the superficial spinal cord. Thus the VGCC $\alpha 2 / \delta-1$ subunit was co-expressed with isolectin B4 (IB4, a marker of non-peptidergic C-type neurons), calcitonin generelated peptide (CGRP) and substance $\mathrm{P}$ (SP, a marker of peptidergic neurons) (Figure 3D). Additionally, $\alpha 2 / \delta$-1-IR cells were observed in neurons of the deep spinal dorsal horn (Figure 3C, 4A).

\section{Expression of CX3CL1 and CX3CR1 in the spinal dorsal horn}

Previous studies suggested that fractalkine (CX3CL1) and its unique CX3CL1 receptor (CX3CR1) play a role in signaling between the neurons and microglia [26-28]. To further address the possible mechanisms by which gabapentin inhibits microglial activation in the spinal cord, we examined the expression of CX3CL1 and CX3CR1 in the spinal dorsal horn. CX3CL1-IR was extensively distributed in all layers of the spinal dorsal horn, including the propriospinal neurons and the primary afferent termination. Additionally, CX3CL1-IR colocalized with the neuronal marker NeuN and the VGCC $\alpha 2 / \delta-1$ subunit (Figure 4A). Following the CFAinduced MA, CX3CL1 expression levels were increased in the ipsilateral spinal dorsal horn, which was suppressed by repeated i.p. injections of gabapentin $(100 \mathrm{mg} / \mathrm{kg})$ (One-way ANOVA, $\mathrm{F}_{2,9}=17.79, \mathrm{q}=7.60$, $\mathrm{p}<0.01$ ) (Figure $4 \mathrm{~B}$ and $4 \mathrm{C}$ ). 

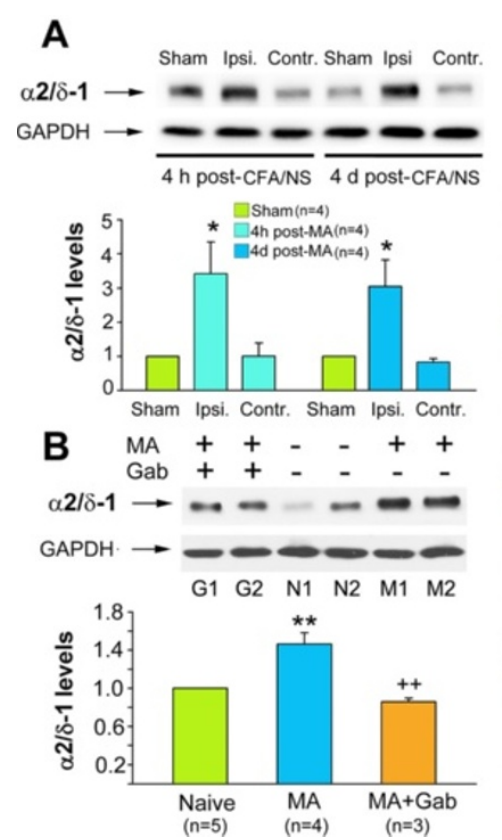
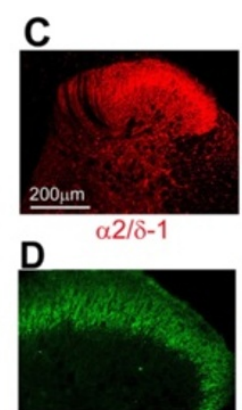

IB4

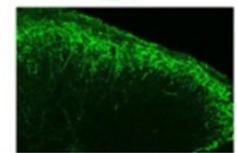

CGRP

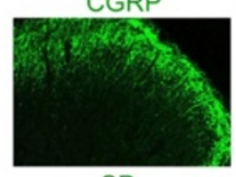

SP

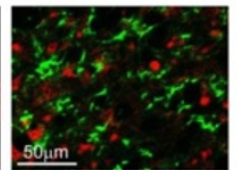

$\alpha 2 / \delta-1 / \mathrm{lba}-1$

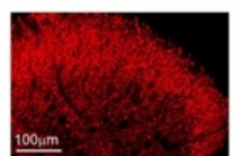

$\alpha 2 / \delta-1$

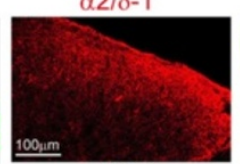

$\alpha 2 / \delta-1$

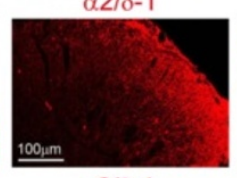

$\alpha 2 / \delta-1$

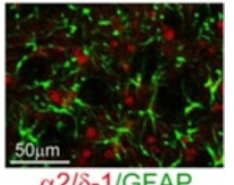

$\alpha 2 / \delta-1 / G F A P$

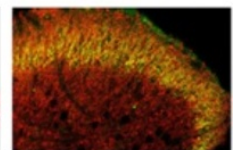

IB4/ $\alpha 2 / 8-1$

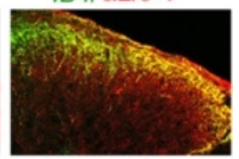

$\mathrm{CGRP} / \alpha 2 / \delta-1$

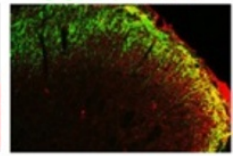

$\mathrm{SP} / \alpha 2 / \delta-1$

Figure 3 Expression of the voltage-gated calcium channels (VGCCs) a $\mathbf{2} / \mathbf{\delta}-\mathbf{1}$ subunit in the lumbar spinal cord. A, The western blot shows an increase in the level of VGCC a2/ $\delta$ - 1 subunit expression in the ipsilateral lumbar spinal dorsal horn at 4 hrs and 4 days after MA (CFA/NS injected into the ankle articular cavity). B, Western blot analysis reveals that repeated Gab treatment significantly suppresses MA-induced upregulation of the VGCC a2/ $\delta$-1 subunit in the ipsilateral lumbar spinal dorsal horn. ${ }^{*} p<0.05$, ${ }^{* *} p<0.01$ vs. control (sham MA or naïve); ++ $\mathrm{p}<0.01$ vs. MA. C, Immunohistochemistry reveals that the VGCC a2/ $\delta-1$ subunits are extensively distributed in the spinal dorsal horn. Double immunofluorescence staining shows that the VGCC a2/ $\delta-1$ subunit does not colocalized with the microglial marker Iba-1 or the astrocytic marker GFAP in the spinal dorsal horn. D, Double immunofluorescence staining shows that VGCC a2/ $\delta$ - 1 subunits are colocalized with IB4 in the inner lamina II and CGRP and SP in the lamina I and outer lamina II of the spinal cord.

CX3CR1-IR was detected in whole layers of the spinal cord. Similar to previous studies in neuropathic pain models [29,30], CX3CR1 was co-expressed with spinal OX-42-IR (another microglial marker) rather than GFAP in CFA-induced MA rats (Figure 4D). Consistent with our previous study [8], CFA induced a significant upregulation of CX3CR1 expression in the bilateral spinal dorsal horn on day 4 after MA. Repeated i.p. injections of gabapentin $(100 \mathrm{mg} / \mathrm{kg})$ caused a decrease in CX3CR1 expression in the spinal cord by day 4 post-MA, although this did not reach statistical significance (One-way ANOVA, $\mathrm{F}_{2,9}=4.439, \mathrm{q}=3.111, \mathrm{p}=0.055$ ) (Figure 4E).

\section{Discussion}

The main findings of the present study are that the VGCCs $\alpha 2 / \delta-1$ subunit (a proposed gabapentin target of action), CX3CL1 and its receptor CX3CR1 (a potential trigger to activate microglia) are upregulated in the spinal cord following intra-articular injection of CFA. These upregulated proteins were associated with the activation of spinal glia and the development of thermal hyperalgesia. Gabapentin treatment attenuated MAinduced thermal hyperalgesia and activation of spinal glia. Gabapentin treatment also decreased expression of the $\alpha 2 / \delta-1$ subunit and CX3CL1. Consistent with thses observations, a previous study from Wodarski et al [24] showed that in STZ-induced diabetic neuropathy mechanical allodynia was associated with increased spinal microglial activation. In this study, gabapentin treatment significantly attenuated established mechanical allodynia and spinal microglial activation.

An early microglial and delayed astrocytic activation in the spinal dorsal horn have been observed in neuropathic and inflammatory pain models [3,5,8-10]. Despite some conflicting reports $[27,30]$, most evidence showed that spinal microglia and astrocytes were activated by intraplantar or intra-articular injection of CFA, subcutaneous injection of phospholipase A2, snake venom and formalin $[8-10,31,32]$. The present results showed that spinal microglia were activated ipsilaterally at $4 \mathrm{hrs}$ and bilaterally at 1 day after MA. Meanwhile, activation of the spinal astrocytes was detected bilaterally at 4 day after MA. Similar to the present study, glial activation is observed bilaterally in the lumbar sections of the spinal cord in other peripheral inflammation models [3,27]. This is in contrast to peripheral neuropathy, which resulted in glial activation that was predominantly localized ipsilateral to the injury [33]. We are unable to give a clear explanation of this phenomenon at the present. The temporal and spatial sequence of microglial and 


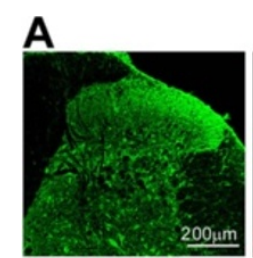

CX3CL1

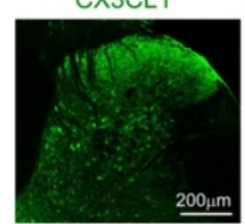

CX3CL1

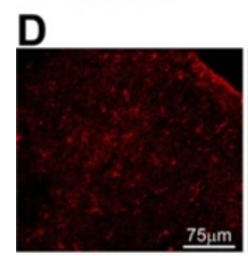

CX3CR1

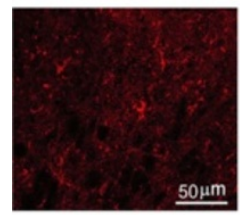

CX3CR1

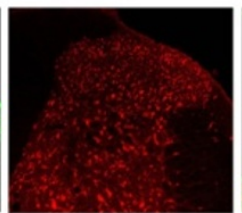

NeuN

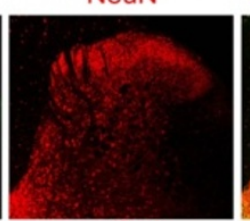

$\alpha_{2} / \delta-1$

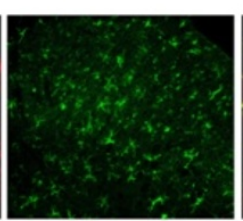

OX-42

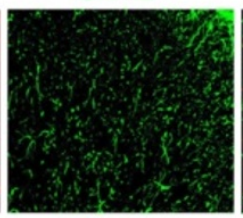

GFAP

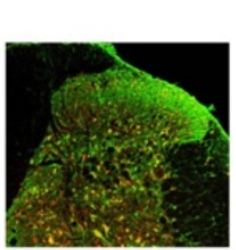

CX3CL1/NeuN

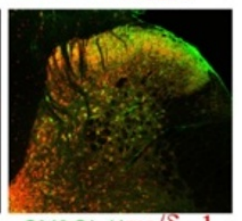

CX3CL $1 / \alpha_{2} / \delta-1$

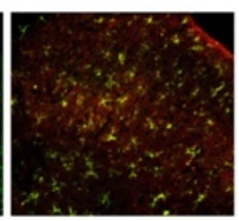

CX3CR1/OX-42

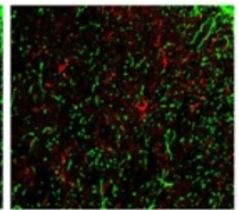

CX3CR1/GFAP
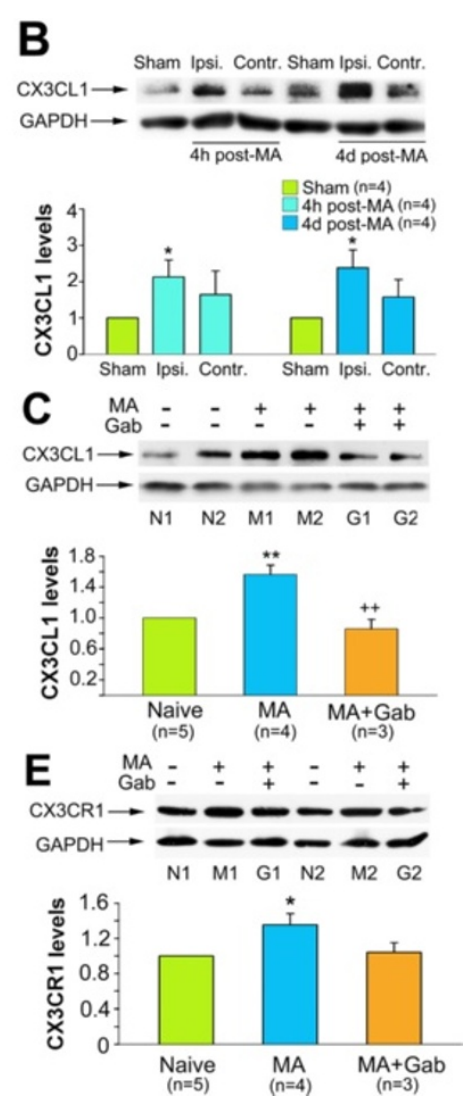

Figure 4 Expression of CX3CL1 and its receptor CX3CR1 in the lumbar spinal cord. A, Double immunofluorescence staining shows that CX3CL1 is colocalized with the neuronal marker NeuN and VGCC a2/ $\delta$-1 subunits in all of the layers of the spinal dorsal horn. B, The western blot shows an increase in the level of CX3CL1 in the ipsilateral lumbar spinal dorsal horn at 4 hrs and 4 days after MA. C, Western blot analysis reveals that repeated Gab treatment significantly suppresses MA-induced upregulation of CX3CL1 in the lumbar spinal dorsal horn. D, Double immunofluorescence staining shows that CX3CR1 is colocalized with the microglial marker OX-42, but not the astrocytic marker GFAP. E, Western blot analysis reveals that repeated Gab treatment partially inhibits MA-induced upregulation of CX3CR1 in the lumbar spinal dorsal horn. ${ }^{*} p<0.05,{ }^{* *} p<0.01$ vs. control (sham MA or naïve); $++P<0.01$ vs. MA.

astrocytic activation in the spinal cord after MA suggest that glialexcitatory substances from the focal ipsilateral microglia response in the early phase (such as $4 \mathrm{hrs}$ after MA in the present study) may spread to the contralateral spinal cord (Figure 5). The proinflammatory cytokines TNF- $\alpha$, IL- $1 \beta$ and IL- 6 are generally considered to play key roles in mediation of this event $[6,34]$. Our previous study demonstrated a bilateral upregulation of TNF- $\alpha$, IL- $1 \beta$ and IL- 6 mRNA by MA [9]. Additionally, astrocytes are organized in gap junction-coupled networks, which could transmit $\mathrm{Ca}^{2+}$ signaling in the form of oscillations or waves through the networks $[35,36]$. Thus, it is possible that astrocyte networks could mediate the spread. Another explanation is that the bilateral glial response to inflammation results from systemic or perivascular signals [37]. It has been demonstrated that in inflammatory pain, there is a partial opening in the tight junctions between the endothelial cells of the capillaries, increasing the blood-brain barrier permeability [38].
It is well known that activated microglia and astrocytes release several proinflammatory cytokines, chemokines, and other neuro- and glial-excitatory substances. These substances facilitate pain processing by further enhancing glial activation, presynaptic release of neurotransmitters and postsynaptic excitability. Such positive feedback loops sustain the persistent release of pain mediators, facilitating the development of neuronal hypersensitivity, which leads to hyperalgesia and allodynia $[1,5,7,13]$. Interestingly, although spinal glial activation was observed in the dorsal horn contralateral to the MA, the contralateral hind paw nociceptive thresholds remained unchanged. Indeed, bilateral increases in neuropeptide synthesis [40] and phosphorylation of the cAMP-responsive element-binding protein (CREB) [41] have been reported in the spinal cord following unilateral injection of CFA and formalin, respectively. These events were shown to produce hyperalgesia ipsilaterally. Additionally, several reports have shown that spinal glial activation and enhanced pain states are disassociated 


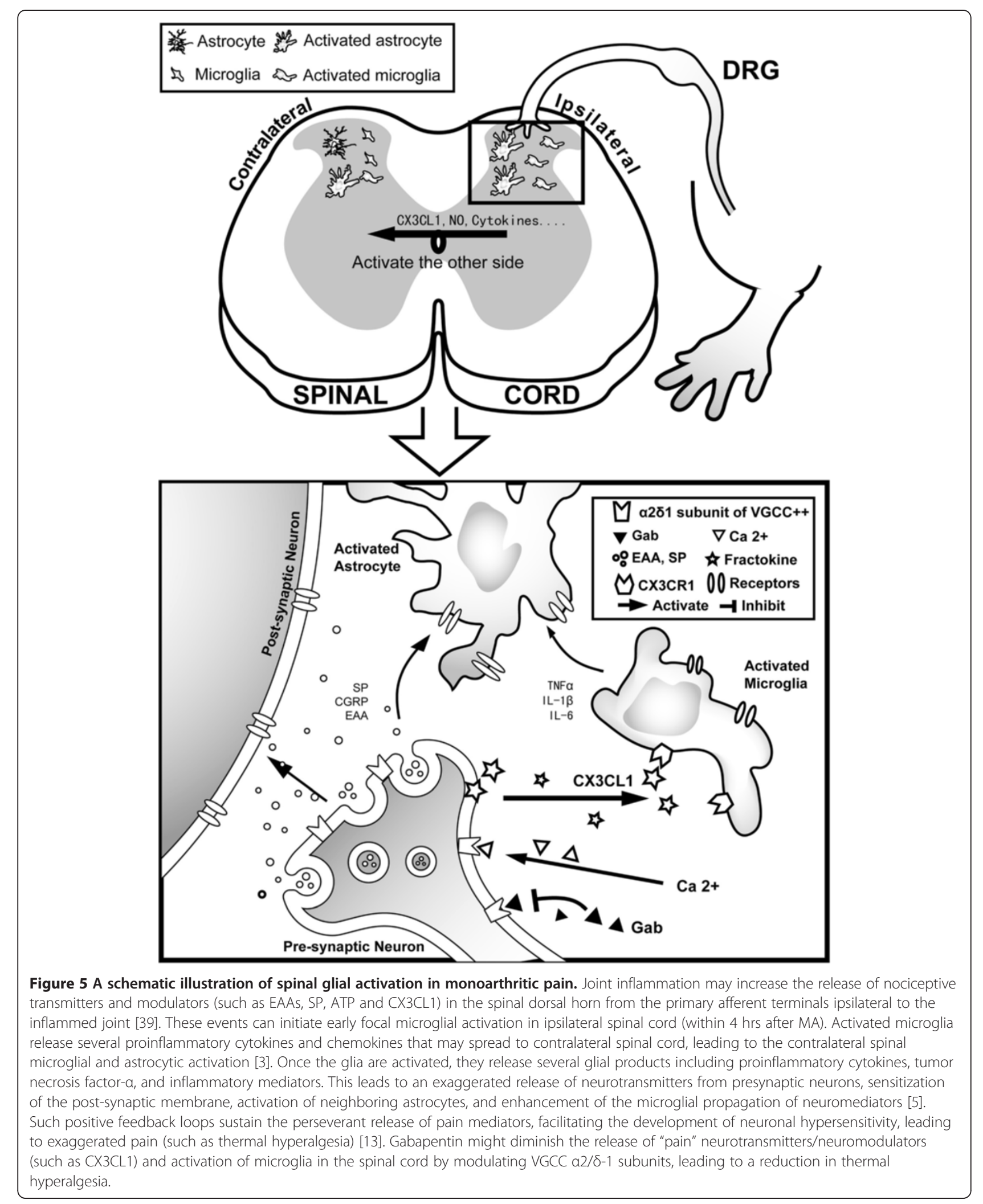


when determined by OX-42 or Iba-1 (microglia) and GFAP (astrocyte) immunoreactivity. For example, Colburn et al. observed that in some rats pain behaviors existed in the apparent absence of microglial activation. Conversely, a profound microglial response was occasionally associated with a lack of pain behaviors [33]. Zhuang et al. showed that a JNK inhibitor reversed neuropathic pain for several days without an accompanying reduction of GFAP expression [42]. Our present study showed that MA resulted in a bilateral upregulation of Iba-1 and GFAP expression in the spinal cord without contralateral thermal hyperalgesia. Additionally, gabapentin attenuated thermal hyperalgesia and Iba-1 expression but did not significantly suppress GFAP expression. Iba- 1 or OX-42 and GFAP are the most widely used markers for demonstrating microglial and astrocytic activation at present. However, whether Iba-1 and GFAP contribute to pain hypersensitivity per se remains unclear. Thus, more sensitive markers of nociceptive changes and glial activation need to be examined in further studies. An alternative explanation for the contralateral side, which did not present obvious hyperalgesia may be that the contralateral hind limb becomes a loadbearing limb when the unilateral knee joint suffers from arthritis. Thus, unilateral load-bearing may affect the measurement of PWLs on free-moving rats.

The glia are sensitive to many types of disturbances in the nervous system. Tissue/nerve inflammation/damage leads to the release of nociceptive transmitters and modulators, including excitatory amino acids (EAAs), CGRP, substance P (SP), ATP and NO from the primary afferent neurons [39]. All of the "pain" neurotransmitters/modulators can cause glial activation via the corresponding receptors expressed on the spinal microglia and astrocytes (such as NMDA, AMPA/KA, NK-1, P2X4 and $\mathrm{P} 2 \mathrm{X} 7$ etc.) [43]. It has been shown that gabapentin is capable of reducing the release of peripheral inflammation or nerve injury-induced spinal EAAs (glutamate and aspartate) [44] and neuropeptides (SP and CGRP) [45]. Gabapentin also inhibits the enhancement of $\mathrm{K}^{+}$- or SPand CGRP-evoked glutamate release in caudal trigeminal slices [46]. Consistent with the inhibition of spinal glutamate release, gabapentin markedly reduces miniature EPSCs in spinal cord slice [47]. Thus, one of the mechanisms by which gabapentin suppresses MA-induced spinal glial activation, might be by diminishing the release of the "pain" neurotransmitters/neuromodulators from the primary afferent terminals and spinal nociceptive neurons. In the present study, the reduction of spinal microglial marker by gabapentin is unlikely due to a direct action on glia because no colocalization of the VGCCs $\alpha 2 / \delta-1$ subunit (a binding target of gabapentin) and Iba-1 (a microglial marker) was found in the spinal dorsal horn. In contrast, double staining signals of the
VGCCs $\alpha 2 / \delta-1$ subunit and IB4 (a marker of nonpeptidergic C-type neurons) or CGRP and SP (markers of peptidergic neurons in superficial spinal dorsal horn) were observed. It was also reported that mRNA levels of the VGCCs $\alpha 2 / \delta-1$ subunit were increased in the DRG in osteoarthritis [48]. This finding is consistent with the distribution of the $\alpha 2 / \delta-1$ subunit in the primary afferent terminals of the spinal cord. It should be mentioned that gabapentin was also reported to block the persistent $\mathrm{Na}^{+}$current in DRG neurons at relatively low concentrations (compared to doses achieved clinically). Importantly, this blockage occurred at a somewhat lower dose than required to inhibit the $\mathrm{Ca}^{2+}$ channel in DRG neurons, suggesting that there is another analgesic action target of gabapentin [22]. This could occur if gabapentin suppressed the abnormal spontaneous activity and DRG hyperexcitability, resulting in an attenuation of spinal glial activation.

Fractalkine (CX3CL1) is a unique chemokine in the central nervous system (CNS), which acts exclusively on the CX3CL1 receptor (CX3CR1) [49]. Intriguingly, both in the spinal cord and dorsal root ganglion (DRG), CX3CL1 immunoreactivity and mRNA were observed in neurons. However, CX3CR1 immunoreactivity and mRNA were found to be mainly restricted to the OX42- and CD4-positive microglia in the spinal dorsal horn $[27,29,30]$. This led to the idea of CX3CL1 as a critical mediator of spinal neuronal-microglial communication in chronic pain [26,28]. Following peripheral nerve injury and inflammation, CX3CR1 knockout mice showed deficits in inflammatory and neuropathic nociceptive responses. In contrast, wild-type mice showed significant thermal hyperalgesia and mechanical allodynia. This was associated with upregulated CX3CL1 and CX3CR1 levels and increased Iba-1 immunostaining as well as phosporylation of p38 MAPK in the spinal cord [50]. Consistent with this data, the present study showed that CX3CL1 immunoreactivity was detected in all layers of the spinal dorsal horn. CX3CL1 also colocalized with the VGCC $\alpha 2 / \delta-1$ subunit and almost all of the CX3CR1-positive cells were OX-42 (a microglial marker)-positive cells. Moreover, following CFA-induced MA, expression of the VGCC $\alpha 2 / \delta-1$ subunit, CX3CL 1 and CX3CR 1 in the spinal dorsal horn were increased. This increase in expression was attenuated by gabapentin. A discrepancy is that MA induced unilateral upregulation of the VGCC $\alpha 2 / \delta-1$ subunit, upregulation of CX3CL1 and bilateral activation of microglia and astrocytes. We presume that peripheral inflammation may increase the release of nociceptive transmitters and modulators (such as EAAs, SP, ATP and CX3CL1) in the spinal dorsal horn. This may occur via the upregulated VGCC $\alpha 2 / \delta-1$ subunits on the primary afferent terminals ipsilateral to inflammatory arthritis. This initiates early focal microglial 
activation in the ipsilateral spinal cord (within $4 \mathrm{hrs}$ after MA). Activated microglia release several proinflammatory cytokines and chemokines that may spread to the contralateral spinal cord, leading to contralateral spinal microglial and astrocytic activation. Actually, a trend towards increased CX3CL1 expression was observed in the spinal cord contralateral to the MA (Figure 4B). Additionally, our previous study revealed a bilateral upregulation of CX3CR1 in the spinal dorsal horn [8]. Preemptive and early consecutive treatments of gabapentin may block microglial activation by modulating VGCC $\alpha 2 / \delta-1$ subunits to attenuate the release of nociceptive transmitters and modulators (including CX3CL1) in the spinal cord (Figure 5).

\section{Conclusions}

Gabapentin treatment attenuated MA-induced thermal hyperalgesia and spinal glial activation. The reduction of spinal glial activation by gabapentin is likely to be due to an indirect action on the glia by modulation of the VGCC $\alpha 2 / \delta-1$ subunits. This modulation may diminish the release of the "pain" neurotransmitters/neuromodulators (including CX3CL1) from primary afferent terminals and spinal nociceptive neurons.

\section{Methods}

\section{Animals}

The experiments were performed on adult male Sprague Dawley rats (Experimental Animal Center, Shanghai Medical College of Fudan University, China) weighing $180-220 \mathrm{~g}$. The animals were housed in a temperaturecontrolled $\left(22 \pm 2^{\circ} \mathrm{C}\right)$ and light-controlled (12-h dark /12-h light cycle) room with free access to food and water. All of the experimental protocols and animal handling procedures were approved by the Animal Care and Use Committee of Fudan University and were consistent with the National Institutes of Health Guide for the Care and Use of Laboratory Animals. All efforts were made to minimize the number of animals used and their suffering.

\section{Drugs}

Gabapentin (Jiangsu Hengrui Medicine CO. LTD) was diluted in normal saline (NS, $0.9 \% \mathrm{NaCl}$ ). It was administered i.p. in a dose of $100 \mathrm{mg} / \mathrm{kg}$ body weight [14]. Control animals received an equivalent volume of sterile NS.

\section{Induction of monoarthritis}

Monoarthritis (MA) was induced by injection of complete Freund's adjuvant (CFA) into the unilateral ankle articular cavity [11]. The rat was briefly anesthetized with isoflurane. The skin around the site of injection was sterilized with iodine tincture and $75 \%$ alcohol.
The right leg of the rat was held and the fossa of the lateral malleolus of the fibula was located. A 28-gauge needle was inserted vertically to penetrate the skin and turned distally to insert into the articular cavity from the gap between the tibiofibular and tarsus bone, until a distinct loss of resistance was felt. A volume of $50 \mu \mathrm{l}$ of CFA was then injected. Sham MA control animals were similarly injected with sterile NS.

\section{Hargreaves' test for thermal hyperalgesia}

After acclimation to the test chamber, thermal hyperalgesia was assessed by measuring the latency of paw withdrawal in response to a radiant heat source. The rats were housed individually in Plexiglas chambers on an elevated glass platform under a radiant heat source (Model 336 Combination Unit, IITC/life Science Instruments, Woodland Hill, CA, USA). The heat was applied to the plantar surface of the hind paw through the glass plate. The heat source was turned off when the rat lifted the foot, allowing measurement of the time from the onset of radiant heat application to withdrawal of the rat's hindpaw. This time was defined as the paw withdrawal latency (PWL). The heat was maintained at a constant intensity, which produced a stable PWL of approximately $10-12 \mathrm{~s}$ in the absence of arthritis. A $20 \mathrm{~s}$ cutoff was used to prevent tissue damage. Both hindpaws were tested independently with a $10 \mathrm{~min}$ interval between trials [8].

\section{Immunohistochemistry}

After defined survival times, rats were given an overdose of urethane $(2 \mathrm{~g} / \mathrm{kg}$, i.p. $)$ and perfused intracardially with saline followed by $4 \%$ paraformaldehyde in $0.1 \mathrm{M}$ phosphate buffer ( $\mathrm{PB}, \mathrm{pH}$ 7.4). The L4/5 segments of the spinal cord were then removed, post-fixed in the same fixative for $4 \mathrm{~h}$ at $4^{\circ} \mathrm{C}$, and immersed from $10 \%$ to $30 \%$ gradient sucrose in PB for $24-48 \mathrm{~h}$ at $4^{\circ} \mathrm{C}$ for cryoprotection. Transverse spinal sections $(35 \mu \mathrm{m})$ were cut in a cryostat and processed for immunofluorescence. All of the sections were blocked with $10 \%$ donkey serum in $0.01 \mathrm{M}$ phosphate buffered saline (PBS, pH 7.4) with $0.3 \%$ Triton $\mathrm{X}-100$ for $2 \mathrm{~h}$ at RT. The samples were then incubated overnight at $4^{\circ} \mathrm{C}$ with rabbit anti-Iba- 1 $(1: 2000$, Serotec), mouse anti-calcium channel $(\alpha 2 / \delta-1$ subunit) (1:500, LifeSpan Biosciences) or rabbit anti-glial fibrillary acidic protein (GFAP, 1:2000, Sigma) primary antibodies in PBS with $1 \%$ normal donkey serum and $0.3 \%$ Triton $\mathrm{X}-100$. Following three $10 \mathrm{~min}$ rinses in $0.01 \mathrm{M}$ PBS, the sections were incubated with fluorescein isothiocyanate (FITC)-conjugated donkey anti-mouse IgG (1:200, Jackson Immunolab) or rhodamine-conjugated donkey anti-rabbit IgG (1:200, Jackson Immunolab) for $120 \mathrm{~min}$ at $4{ }^{\circ} \mathrm{C}$, then washed with PBS. For the $\alpha 2 / \delta-1 /$ GFAP, $\alpha 2 / \delta-1 / \mathrm{Iba}-1, \alpha 2 / \delta-1 / \mathrm{CGPR}$, 
$\alpha 2 / \delta-1 /$ IB4 $4, \alpha 2 / \delta-1 / \mathrm{SP}, \alpha 2 / \delta-1 / \mathrm{CX} 3 \mathrm{CL} 1, \mathrm{CX} 3 \mathrm{CR} 1 / \mathrm{OX}-42$ and CX3CR1/GFAP double immunofluorescence, the sections were incubated with a appropriate mixtures of the following antibodies overnight at $4^{\circ} \mathrm{C}$ : mouse anti$\alpha 2 / \delta-1$ and rabbit anti-GFAP, rabbit anti-Iba- 1 , goat anti-CGRP (1:500, Santa Cruze), rabbit anti-SP (1:100, Sigma), goat anti-CX3CL1 (1:200, R\&D system), or rabbit anti-CX3CR1 (1:1000, Abcampare; goat antiCX3CR1, 1:2000, Santa Cruz Biotechnology) and mouse anti-NeuN (1:5000, Millipore), mouse anti-OX-42 (1:3000, Serotec), or mouse anti-GFAP (1:2000, Sigma). This was followed by incubation with a mixture of FITC- and rhodamine red-X-conjugated secondary antibodies or FITC-conjugated IB4 (1:1000, Sigma) for $2 \mathrm{~h}$ at RT. The specificity of immunostaining was verified by omitting the primary antibodies. The specificity of the primary antibodies was verified by the preabsorption experiment (control peptide for CX3CL1 from Acris Antibodies; for CX3CR1 from Abcampare and Santa Cruz; for $\alpha 2 / \delta-1$ from LifeSpan). All of the sections were coverslipped with a mixture of $50 \%$ glycerin in $0.01 \mathrm{M}$ PBS, and then observed with a Leica SP2 confocal laserscanning microscope. Because the morphology of both microglia and astrocytes is complex and immunoreactive staining includes both cell bodies and their processes, cell counts may not sufficiently quantify activation. Therefore, the optical density of immunoreactive staining for Iba-1 or GFAP was measured with the Image J analysis system [51]. For each animal, six to eight sections of spinal L4-5 sections were randomly selected for quantitative evaluation. The corrected density values of the sections were averaged to provide a mean density for each animal.

\section{Western blot analysis}

After defined survival times, rats were killed by an overdose of urethane (SCR Co., Shanghai, China). The L4-5 spinal cord from naive, MA or gabapentin-treated rats was rapidly removed. Then the spinal segment was cut into a left and right half from the ventral midline. Finally, the right half was further split into the dorsal and ventral horn at the level of the central canal. After dissection, all of the tissues were rapidly frozen in liquid nitrogen and stored at $-70^{\circ} \mathrm{C}$ until further processing.

Frozen spinal cords were directly homogenized in a lysis buffer $(12.5 \mathrm{ml} / \mathrm{mg}$ tissue) containing a cocktail of protease inhibitors and PMSF (Sigma). After centrifugation at 10,000 rpm for $15 \mathrm{~min}$, the supernatant was used for western blot analysis. Equal amounts of protein $(20 \mathrm{mg})$ were loaded into each lane and separated by 10\% SDS-PAGE. The resolved proteins were transferred onto PVDF membranes. The membranes were blocked in $10 \%$ nonfat dry milk for $2 \mathrm{~h}$ at room temperature (RT). The membranes were then incubated overnight at $4^{\circ} \mathrm{C}$ with mouse anti- $\alpha 2 / \delta-1$ (1:100), goat anti-CX3CL1 (1:1000), rabbit anti-CX3CR1 (1:1000) or mouse antiGAPDH (1:10000, Sigma) primary antibodies. The blots were incubated for $2 \mathrm{~h}$ at RT with horseradish peroxidase (HRP)-conjugated donkey anti-mouse, donkey antigoat or donkey anti-rabbit secondary antibodies (1:5000, Santa Cruz Biotechnology). The signals were visualized using enhanced chemiluminescence (ECL, Pierce) and exposed onto X-films for 1-10 min. Pre-absorption of the primary antibodies with the blocking peptide served as specificity control. All western blot analyses were performed at least three times, and parallel results were obtained. X-ray films with blotting bands for each sample from different rats were scanned, and the density of the band area was quantified with a method described by Sun et al. [8]. The same size square was drawn around each band to measure the density, and the background near that band was subtracted. GAPDH expression was used as a loading control for protein expression. The expression level of the proteins is an average of the densities per band area from different rats that were treated.

All of the behavioral testing and quantification of immunohistochemical and western blot experiments were performed blindly, with respect to the treatments.

\section{Statistical analysis}

The data are given as the mean \pm standard error of the mean (S.E.M.). Both the pre-MA baseline and pre-drug treatment measures were analyzed by a one-way analysis of variance (ANOVA). The post-drug time course measures for hyperalgesia were analyzed by a two-way RM ANOVA (treatment $\times$ time) followed by a NewmanKeuls post hoc test. Immunohistochemical and western blot analysis were performed using Student's t-test when comparing two groups, or a one-way ANOVA followed by Dunnett's test for multiple comparisons when comparing more than two groups. A value of $p<0.05$ was considered statistically significant.

\section{Experimental design}

Experiment 1 was designed to test the effects of repeated i.p. injection of gabapentin on MA-induced thermal hyperalgesia. After the baseline behavioral assessments, the rats received an intra-articular injection of $50 \mu \mathrm{l}$ CFA or sterile NS (day 0). Repeated i.p. injections of gabapentin $(100 \mathrm{mg} / \mathrm{kg})$ or vehicle (NS) were carried out once daily for 4 days after behavioral testing. The first injection was given $60 \mathrm{~min}$ before the intra-articular injection of CFA. A Hargreaves' test was performed from days 1 to 6. Experiment 2 was designed to test MA-induced changes in spinal GFAP, Iba-1, VGCC $\alpha 2 / \delta-1$ subunit and CX3CL1 expression levels. Rats received an intraarticular injection of $50 \mu \mathrm{l} \mathrm{CFA}$ or sterile NS. The rats 
were then sacrificed at 4 hrs, 1 and 4 days after CFA injection for immunohistochemical or western blot processing. Experiment 3 was designed to test the effects of repeated i.p. injection of gabapentin on MA-induced changes in GFAP, Iba-1, VGCC $\alpha 2 / \delta-1$ subunit, CX3CL1, and CX3CR1 expression levels in the lumbar spinal cord.

\section{Competing interests}

The authors do not have any competing interest to declare.

\section{Authors' contributions}

Yang $J \mathrm{~L}$ and $\mathrm{Xu} \mathrm{B}$ performed the behavioral tests, western blot and immunofluorescence experiments and drafted the manuscript. Li SS participated in the behavioral tests. Zhang WS, Xu H and Deng XM participated in producing the statistical analysis and experimental design. Zhang YQ conceived the study, designed the experiments, wrote the paper and coordinated the authors. All of the authors read and approved the final manuscript.

\section{Acknowledgements}

This work was supported by National Natural Science Fund of China (NSFC, 31121061,81070895 and 31070973). None of the authors report any competing interests in this study.

\section{Author details}

${ }^{1}$ Institute of Neurobiology, Institutes of Brain Science and State Key Laboratory of Medical Neurobiology, Fudan University, Shanghai 200032, China. ${ }^{2}$ Department of Anesthesiology, Xinhua Hospital, Shanghai Jiaotong University School of Medicine, Shanghai 200092, China. ${ }^{3}$ Department of Anesthesiology, Changhai Hospital, The Second Military Medical University, Shanghai 200433, China.

Received: 27 February 2012 Accepted: 5 April 2012

Published: 30 May 2012

\section{References}

1. Watkins $L R$, Milligan ED, Maier SF: Glial activation: a driving force for pathological pain. Trends Neurosci 2001, 24:450-455.

2. Tsuda M, Inoue K, Salter MW: Neuropathic pain and spinal microglia: a big problem from molecules in "small" glia. Trends Neurosci 2005, 28:101-107.

3. Cao H, Zhang YQ: Spinal glial activation contributes to pathological pain states. Neurosci Biobehav Rev 2008, 32:972-983.

4. Twining CM, Sloane EM, Schoeniger DK, Milligan ED, Martin D, Marsh H, Maier SF, Watkins LR: Activation of the spinal cord complement cascade might contribute to mechanical allodynia induced by three animal models of spinal sensitization. J Pain 2005, 6:174-183.

5. Gwak YS, Kang J, Unabia GC, Hulsebosch CE: Spatial and temporal activation of spinal glial cells: Role of gliopathy in central neuropathic pain following spinal cord injury in rats. Exp Neurol 2011, 2011.10.010. [Epub ahead of print]

6. Milligan ED, Twining C, Chacur M, Biedenkapp J, O'Connor K, Poole S, Tracey K, Martin D, Maier SF, Watkins LR: Spinal glia and proinflammatory cytokines mediate mirror-image neuropathic pain in rats. J Neurosci 2003, 23:1026-1040.

7. Ledeboer A, Sloane EM, Milligan ED, Frank MG, Mahony JH, Maier SF, Watkins LR: Minocycline attenuates mechanical allodynia and proinflammatory cytokine expression in rat models of pain facilitation. Pain 2005, 115:71-83.

8. Sun $\mathrm{S}, \mathrm{Cao} H$, Han M, Li TT, Pan HL, Zhao ZQ, Zhang YQ: New evidence for the involvement of spinal fractalkine receptor in pain facilitation and spinal glial activation in rat model of monoarthritis. Pain 2007, 129:64-75.

9. Sun S, Mao-Ying QL, Cao H, Li TT, Han M, Pan HL, Wang YQ, Zhao ZQ, Zhang YQ: Functional state of spinal microglia involved in the antiallodynic and anti-hyperalgesic effects of electroacupuncture in rat model of monoarthritis. Neurobiol Dis 2007, 26:558-568.

10. Sun S, Cao H, Han M, Li TT, Zhao ZQ, Zhang YQ: Evidence for suppression of electroacupuncture on spinal glial activation and behavioral hypersensitivity in a rat model of monoarthritis. Brain Res Bull 2008, 75:83-93.
11. Sun $S$, Chen WL, Wang PF, Zhao ZQ, Zhang YQ: Disruption of glial function enhances electroacupuncture analgesia in arthritic rats. Exp Neurol 2006, 198:294-302.

12. Segal AZ, Rordorf G: Gabapentin as a novel treatment for postherpetic neuralgia. Neurology 1996, 46:1175-1176.

13. Hulsebosch CE, Xu GY, Perez-Polo JR, Westlund KN, Taylor CP, McAdoo DJ: Rodent model of chronic central pain after spinal cord contusion injury and effects of gabapentin. J Neurotrauma 2000, 17:1205-1217.

14. Cheng JK, Chiou LC: Mechanisms of the antinociceptive action of gabapentin. J Pharmacol Sci 2006, 100:471-486.

15. Zhang WS, Xu H, Xu B, Sun S, Deng XM, Zhang YQ: Antihyperalgesic effect of systemic dexmedetomidine and gabapentin in a rat model of monoarthritis. Brain Res 2009, 1264:57-66.

16. Vonsy JL, Ghandehari J, Dickenson AH: Differential analgesic effects of morphine and gabapentin on behavioural measures of pain and disability in a model of osteoarthritis pain in rats. Eur J Pain 2009, 13:786-793.

17. Luo ZD, Chaplan SR, Higuera ES, Sorkin LS, Stauderman KA, Williams ME, Yaksh TL: Upregulation of dorsal root ganglion (alpha)2(delta) calcium channel subunit and its correlation with allodynia in spinal nerve-injured rats. J Neurosci 2001, 21:1868-1875.

18. Field MJ, Cox PJ, Stott E, Melrose H, Offord J, Su TZ, Bramwell S, Corradini L, England S, Winks J, Kinloch RA, Hendrich J, Dolphin AC, Webb T, Williams D: Identification of the alpha2-delta-1 subunit of voltage-dependent calcium channels as a molecular target for pain mediating the analgesic actions of pregabalin. Proc Natl Acad Sci USA 2006, 103:17537-17542.

19. Sutton KG, Martin DJ, Pinnock RD, Lee K, Scott RH: Gabapentin inhibits high-threshold calcium channel currents in cultured rat dorsal root ganglion neurones. Br J Pharmacol 2002, 135:257-265.

20. Hendrich J, Van Minh AT, Heblich F, Nieto-Rostro M, Watschinger K, Striessnig J, Wratten J, Davies A, Dolphin AC: Pharmacological disruption of calcium channel trafficking by the alpha2delta ligand gabapentin. Proc Natl Acad Sci USA 2008, 105:3628-3633.

21. Bauer CS, Nieto-Rostro M, Rahman W, Tran-Van-Minh A, Ferron L, Douglas L, Kadurin I, Sri Ranjan Y, Fernandez-Alacid L, Millar NS, Dickenson AH, Lujan R, Dolphin AC: The increased trafficking of the calcium channel subunit alpha2delta- 1 to presynaptic terminals in neuropathic pain is inhibited by the alpha2delta ligand pregabalin. $J$ Neurosci 2009, 29:4076-4088.

22. Yang RH, Wang WT, Chen JY, Xie RG, Hu SJ: Gabapentin selectively reduces persistent sodium current in injured type-A dorsal root ganglion neurons. Pain 2009, 143:48-55.

23. Mixcoatl-Zecuatl T, Medina-Santillán R, Reyes-García G, Vidal-Cantú GC, Granados-Soto V: Effect of $\mathrm{K}+$ channel modulators on the antiallodynic effect of gabapentin. Eur J Pharmacol 2004, 484:201-208.

24. Wodarski R, Clark AK, Grist J, Marchand F, Malcangio M: Gabapentin reverses microglial activation in the spinal cord of streptozotocininduced diabetic rats. Eur J Pain 2009, 13:807-811.

25. Xiao W, Boroujerdi A, Bennett GJ, Luo ZD: Chemotherapy-evoked painful peripheral neuropathy: analgesic effects of gabapentin and effects on expression of the alpha-2-delta type-1 calcium channel subunit. Neuroscience 2007, 144:714-720.

26. Harrison JK, Jiang Y, Chen S, Xia Y, Maciejewski D, McNamara RK, Streit WJ, Salafranca MN, Adhikari S, Thompson DA, Botti P, Bacon KB, Feng L: Role for neuronally derived fractalkine in mediating interactions between neurons and CX3CR1-expressing microglia. Proc Natl Acad Sci USA 1998, 95:10896-10901.

27. Clark AK, Gentry C, Bradbury EJ, McMahon SB, Malcangio M: Role of spinal microglia in rat models of peripheral nerve injury and inflammation. Eur J Pain 2007, 11:223-230.

28. Milligan ED, Sloane EM, Watkins LR: Glia in pathological pain: a role for fractalkine. J Neuroimmunol 2008, 198:113-120.

29. Verge GM, Milligan ED, Maier SF, Watkins LR, Naeve GS, Foster AC: Fractalkine (CX3CL1) and fractalkine receptor (CX3CR1) distribution in spinal cord and dorsal root ganglia under basal and neuropathic pain conditions. Eur J Neurosci 2004, 20:1150-1160.

30. Lindia JA, McGowan E, Jochnowitz N, Abbadie C: Induction of CX3CL1 expression in astrocytes and CX3CR1 in microglia in the spinal cord of a rat model of neuropathic pain. J Pain 2005, 6:434-438. 
31. Watkins LR, Martin D, Ulrich P, Tracey KJ, Maier SF: Evidence for the involvement of spinal cord glia in subcutaneous formalin induced hyperalgesia in the rat. Pain 1997, 71:225-235.

32. Raghavendra V, Tanga FY, DeLeo JA: Complete Freunds adjuvant-induced peripheral inflammation evokes glial activation and proinflammatory cytokine expression in the CNS. Eur J Neurosci 2004, 20:467-473.

33. Colburn RW, DeLeo JA, Rickman AJ, Yeager MP, Kwon P, Hickey WF: Dissociation of microglial activation and neuropathic pain behaviors following peripheral nerve injury in the rat. J Neuroimmunol 1997, 79:163-175.

34. Andersson AK, Rönnbäck L, Hansson E: Lactate induces tumour necrosis factor-alpha, interleukin- 6 and interleukin-1beta release in microglialand astroglial-enriched primary cultures. J Neurochem 2005, 93:1327-1333.

35. Blomstrand F, Aberg ND, Eriksson PS, Hansson E, Rönnbäck L: Extent of intercellular calcium wave propagation is related to gap junction permeability and level of connexin- 43 expression in astrocytes in primary cultures from four brain regions. Neuroscience 1999, 92:255-265.

36. Haydon PG, GLIA: listening and talking to the synapse. Nat Rev Neurosci 2001, 2:185-193.

37. Elmquist JK, Breder CD, Sherin JE, Scammell TE, Hickey WF, Dewitt D, Saper CB: Intravenous lipopolysaccharide induces cyclooxygenase 2-like immunoreactivity in rat brain perivascular microglia and meningeal macrophages. J Comp Neurol 1997, 381:119-129.

38. Huber JD, Witt KA, Hom S, Egleton RD, Mark KS, Davis TP: Inflammatory pain alters blood-brain barrier permeability and tight junctional protein expression. Am J Physiol Heart Circ Physiol 2001, 280:H1241-H1248.

39. Westlund KN, Kochukov MY, Lu Y, McNearney TA: Impact of central and peripheral TRPV1 and ROS levels on proinflammatory mediators and nociceptive behavior. Mol Pain 2010, 6:46.

40. Donaldson LF, McQueen DS, Seckl JR: Neuropeptide gene expression and capsaicin-sensitive primary afferents: maintenance and spread of adjuvant arthritis in the rat. $J$ Physio 1995, 486:473-482.

41. Ji RR, Rupp F: Phosphorylation of transcription factor CREB in rat spinal cord after formalin-induced hyperalgesia: relationship to c-fos induction. J Neurosci 1997, 17:1776-1785.

42. Zhuang ZY, Wen YR, Zhang DR, Borsello T, Bonny C, Strichartz GR, Decosterd I, Ji RR: A peptide c-Jun N-terminal kinase (JNK) inhibitor blocks mechanical allodynia after spinal nerve ligation: respective roles of JNK activation in primary sensory neurons and spinal astrocytes for neuropathic pain development and maintenance. J Neurosci 2006, 26:3551-3560.

43. Raghavendra $V$, Rutkowski MD, DeLeo JA: The role of spinal neuroimmune activation in morphine tolerance/hyperalgesia in neuropathic and shamoperated rats. J Neurosci 2002, 22:9980-9989.

44. Coderre TJ, Kumar N, Lefebvre CD, Yu JS: A comparison of the glutamate release inhibition and anti-allodynic effects of gabapentin, lamotrigine, and riluzole in a model of neuropathic pain. J Neurochem 2007, 100:1289-1299.

45. Fehrenbacher JC, Taylor CP, Vasko MR: Pregabalin and gabapentin reduce release of substance $P$ and CGRP from rat spinal tissues only after inflammation or activation of protein kinase C. Pain 2003, 105:133-141.

46. Maneuf YP, Hughes J, McKnight AT: Gabapentin inhibits the substance P-facilitated $\mathrm{K}(+)$-evoked release of $[(3) \mathrm{H}]$ glutamate from rat caudial trigeminal nucleus slices. Pain 2001, 93:191-196.

47. Patel MK, Gonzalez MI, Bramwell S, Pinnock RD, Lee K: Gabapentin inhibits excitatory synaptic transmission in the hyperalgesic spinal cord. $\mathrm{Br} J$ Pharmacol 2000, 130:1731-1734.

48. Rahman W, Bauer CS, Bannister K, Vonsy JL, Dolphin AC, Dickenson AH: Descending serotonergic facilitation and the antinociceptive effects of pregabalin in a rat model of osteoarthritic pain. Mol Pain 2009, 5:45.

49. Chapman GA, Moores K, Harrison D, Campbell CA, Stewart BR, Strijbos PJ: Fractalkine cleavage from neuronal membranes represents an acute event in the inflammatory response to excitotoxic brain damage. $J$ Neurosci 2000, 20:RC87.
50. Staniland AA, Clark AK, Wodarski R, Sasso O, Maione F, D'Acquisto F, Maicangio M: Reduced inflammatory and neuropathic pain and decreased spinal microglial response in fractalkine receptor (CX3CR1) knockout mice. J Neurochem 2010, 114:1143-1157.

51. Xu B, Zhang WS, Yang JL, Xu H, Deng XM, Zhang YQ: Dexmedetomidine blocks thermal hyperalgesia and spinal glial activation in rat model of monoarthritis. Acta Pharmacol Sin 2010, 31:1-8.

doi:10.1186/1756-6606-5-18

Cite this article as: Yang et al:: Gabapentin reduces $\mathrm{CX} 3 \mathrm{CL} 1$ signaling and blocks spinal microglial activation in monoarthritic rats. Molecular Brain 2012 5:18.

\section{Submit your next manuscript to BioMed Central and take full advantage of:}

- Convenient online submission

- Thorough peer review

- No space constraints or color figure charges

- Immediate publication on acceptance

- Inclusion in PubMed, CAS, Scopus and Google Scholar

- Research which is freely available for redistribution
C) Biomed Central 